\section{Variation in rod and cone density from the fovea to the mid- periphery in healthy human retinas using adaptive optics scanning laser ophthalmoscopy}

EM Wells-Gray ${ }^{1}$, SS Choi ${ }^{1}$, A Bries ${ }^{1,2}$ and N Doble ${ }^{1}$

\begin{abstract}
Purpose To characterize the rod and cone photoreceptor mosaic at retinal locations spanning the central $60^{\circ}$ in vivo using adaptive optics scanning laser ophthalmoscopy (AO-SLO) in healthy human eyes.

Methods AO-SLO images $\left(0.7 \times 0.9^{\circ}\right)$ were acquired at $680 \mathrm{~nm}$ from 14 locations from $30^{\circ}$ nasal retina (NR) to $30^{\circ}$ temporal retina (TR) in 5 subjects. Registered averaged images were used to measure rod and cone density and spacing within $60 \times 60 \mu \mathrm{m}$ regions of interest. Voronoi analysis was performed to examine packing geometry at all locations. Results Average peak cone density near the fovea was $164000 \pm 24000$ cones $/ \mathrm{mm}^{2}$ and decreased to $6700 \pm 1500$ and $5400 \pm 700$ cones $/ \mathrm{mm}^{2}$ at $30^{\circ} \mathrm{NR}$ and $30^{\circ} \mathrm{TR}$, respectively. Cone-to-cone spacing increased from $2.7 \pm 0.2 \mu \mathrm{m}$ at the fovea to $14.6 \pm 1.4 \mu \mathrm{m}$ at $30^{\circ} \mathrm{NR}$ and $16.3 \pm 0.7 \mu \mathrm{m}$ at $30^{\circ} \mathrm{TR}$. Rod density peaked at $25^{\circ} \mathrm{NR}\left(124000 \pm 20000 \mathrm{rods} / \mathrm{mm}^{2}\right)$ and $20^{\circ}$ TR $\left(120000 \pm 12000 \mathrm{rods} / \mathrm{mm}^{2}\right)$ and decreased at higher eccentricities. Center-to-center rod spacing was lowest nasally at $25^{\circ}(2.1 \pm 0.1 \mu \mathrm{m})$. Temporally, rod spacing was lowest at $20^{\circ}(2.2 \pm 0.1 \mu \mathrm{m})$ before increasing to $2.3 \pm 0.1 \mu \mathrm{m}$ at $30^{\circ} \mathrm{TR}$. Conclusions Both rod and cone densities showed good agreement with histology and prior AO-SLO studies. The results demonstrate the ability to image at higher retinal eccentricities than reported previously. This has clinical importance in diseases that initially affect the peripheral retina such as retinitis pigmentosa.
\end{abstract}

Eye (2016) 30, 1135-1143; doi:10.1038/eye.2016.107; published online 27 May 2016

\section{Introduction}

Being the first element in the photo-transduction cascade that triggers vision, the structure and distribution of photoreceptors have long been of interest to clinicians and vision scientists.

Typically, histological studies have been conducted to examine characteristics of the photoreceptor mosaic including total cell count, density, spacing, and size, ${ }^{1-5}$ as well as how these parameters vary with factors such as age ${ }^{6-9}$ and gender. ${ }^{10}$ Results of these investigations vary widely, and the differences may, in part, be due to normal inter-subject variations in cell packing or different population demographics. However, histological analysis can also suffer from specimen preparation artifacts that may systematically skew results.

Cellular level in vivo imaging of the retina can be achieved through the application of adaptive optics (AO) imaging techniques, which have been successfully applied to improve the resolution of a range of imaging modalities, including fundus imaging, ${ }^{11}$ scanning laser ophthalmoscopy (SLO), ${ }^{12}$ and optical coherence tomography (OCT). ${ }^{13,14}$ Compared with ex vivo histological analysis, AO imaging is advantageous in allowing repeatable measurements that can be used to monitor retinal changes over time. To date, the majority of $\mathrm{AO}$ imaging studies have generally been limited to the central $\pm 15^{\circ}$; however, to detect the earliest signs of diseases such as retinitis
${ }^{1}$ College of Optometry, The Ohio State University, Columbus, OH, USA

${ }^{2}$ Department of Biomedical Engineering, The Ohio State University, Columbus, OH, USA

Correspondence: N Doble, College of Optometry, The Ohio State University, 338 West 10th Ave, Columbus, $\mathrm{OH} 43210$, USA

Tel: +1 614688 2052;

Fax: +1614292 1873 .

E-mail: doble.2@osu.edu

Received: 11 December 2015

Accepted in revised form: 14 April 2016 Published online: 27 May 2016 
pigmentosa (RP) and cone-rod dystrophy, ${ }^{15-17}$ requires imaging single cells in the mid-periphery (defined here as $>20^{\circ}$ from the fovea), a capability that has not previously been demonstrated.

Although a number of AO-SLO studies have looked at cone and, more recently, rod distributions of the central retina, ${ }^{18-21}$ studies imaging beyond $15^{\circ}$ from the fovea are relatively few. Cone density as a function of refractive error has been described by Chui et al ${ }^{22}$ out to $12^{\circ}$ in all four meridians. Song et $a l^{23}$ undertook a similar study but examined the cone density variation with age. Dubra et $a l^{19}$ reported on cone density at $5-15^{\circ}$ in the temporal retina (TR) only, while Merino et $a l^{20}$ measured cone spacing out to $12^{\circ}$ in the nasal and inferior retina. Stiles-Crawford effect of the First Kind studies presenting cone images have been reported out to $20^{\circ}$ eccentricities. ${ }^{24,25}$ Reports of in vivo rod measurements are more sparse. Doble et $a l^{26}$ reported rod spacing for $5^{\circ}$ and $10^{\circ}$ in the TR, and the aforementioned studies by Dubra et $a l^{19}$ and Merino et $a l^{20}$ included rod measurements out to $15^{\circ}$ and $12^{\circ}$, respectively. Scoles et $a l^{27}$ showed rod images at $20^{\circ}$ $\mathrm{TR}$, though did not provide quantitative measurements on their distribution or dimensions. Several studies have reported rod densities for various retinal conditions including Stargardt disease, ${ }^{28}$ acute macular neuroretinopathy, ${ }^{29}$ congenital stationary night blindness, ${ }^{30}$ Oguchi disease, ${ }^{30}$ achromatopsia, ${ }^{20}$ and acute zonal occult outer retinopathy. ${ }^{20}$ The results from these studies are all within $12^{\circ}$ of the fovea.

The purpose of this study was to characterize cone and rod photoreceptor density, spacing, and packing geometry at retinal eccentricities out to $30^{\circ}$ in both the TR and nasal retina (NR) in a healthy human population in vivo using an AO-SLO.

\section{Materials and methods}

\section{Subjects}

Five healthy subjects (denoted N1-N5) between the ages of 22 and 27 years were imaged. All subjects underwent a conventional eye examination, including slit lamp examination and ophthalmoscopy. Subjects included three emmetropes (spherical equivalent (SE): +0.25 to -0.75 D; N1-N3), one mild myope (SE: - 2.50 D; N4), and one moderate myope (SE: - 3.75 D; N5). Axial length was measured with a Lenstar LS900 Optical Biometer (HaagStreit, Koniz, Switzerland). Prior to imaging, subjects were dilated with $1 \%$ tropicamide and $2.5 \%$

phenylephrine. A bitebar was used during imaging to minimize head motion. The tenets of the Declaration of Helsinki Principle were observed and the protocol was approved by the Institutional Review Board of The Ohio State University. Written informed consent was obtained after all procedures were fully explained to the subjects and prior to experimental measurements.

\section{AO-SLO imaging}

Subjects were imaged using an AO-SLO system, the design of which is identical to the SLO sub-system of our combined AO-SLO-OCT system. ${ }^{31}$ Briefly, the AO-SLO imaging and wavefront sensor use the same $680 \mathrm{~nm}$ light source (BroadLighter T-680-HP, Superlum, Cork, Ireland), the field of view on the retina is $0.7 \times 0.9^{\circ}(\sim 200 \times 260 \mu \mathrm{m})$ and it is designed to image over a $7.15 \mathrm{~mm}$ exit pupil. A $16 \mathrm{kHz}$ resonant scanner mirror scans the beam horizontally, and a $30 \mathrm{~Hz}$ galvo mirror scans vertically, yielding a $30 \mathrm{~Hz}$ frame rate. A confocal pinhole of diameter equal to $\sim 1$ Airy disk is placed prior to a photomultiplier tube detector (H7422-20, Hamamatsu, Shizuoka, Japan). To correct ocular aberrations, the system uses a high-speed 97-actuator continuous-surface magnetic-membrane deformable mirror (DM; DM97-15, ALPAO, Montbonnot, France) in combination with a Shack-Hartmann wavefront sensor (SHSCam AR-S-150GE, Optocraft, Erlangen, Germany). Imaging power was $100 \mu \mathrm{W}$, well below ANSI limits. ${ }^{32}$ Light exposure was further limited by utilizing an acousto-optic light modulator that switches off the imaging beam for half the period of the resonant scanner (a 50\% duty cycle, yielding $50 \mu \mathrm{W}$ average power) and by limiting time in the system to $30 \mathrm{~s}$ increments.

Subject's right eyes were imaged at the fovea, $3^{\circ} \mathrm{NR}$ and TR, and in $5^{\circ}$ increments from 5 to $30^{\circ}$ on both the NR and TR sides. Imaging at $15^{\circ} \mathrm{NR}$ was excluded due to proximity of the optic nerve head. For each location, the subject fixated on a Maltese cross target displayed on a computer monitor visible through a pellicle beam splitter. The focal plane was scanned axially through the photoreceptor layer in $5 \mu \mathrm{m}$ steps by applying a defocus offset to the DM. Total depth scanned was $\sim 50 \mu \mathrm{m}$ at each location with 100-200 frames acquired for each step. At larger retinal eccentricities, the imaging pupil became progressively elliptical with the effective aperture varying with the cosine of the angle. All subjects had dilated pupil sizes $>8 \mathrm{~mm}$, so this did not present a problem.

Additional cylindrical trial lenses were added as the eccentricity increased to compensate for the increasing astigmatism.

\section{Offline post processing}

Due to warping in the horizontal direction caused by the sinusoidal resonant scanner motion, all frames were dewarped based on a Ronchi ruling calibration image. For each retinal location, $\sim 50$ de-warped frames underwent a strip-wise registration algorithm to correct for eye motion 
and then averaged to increase the signal-to-noise ratio. ${ }^{33,34}$ All post processing was performed using custom-written Matlab routines (MathWorks, Natick, MA, USA).

\section{Cone and rod identification}

Registered images were reviewed to find the focal plane where the rod mosaic appeared brightest. An automated Matlab routine identified all cells over user-selected regions of interest (ROIs). The ROIs were specifically chosen over areas where the photoreceptor mosaic was well resolved and continuous without vasculature. Excluding the fovea, average ROI size was $\sim 60 \times 60 \mu \mathrm{m}$. Near the fovea, where a small sampling window is necessary for accurate cell density measurements, the average ROI was $\sim 35 \times 35 \mu \mathrm{m}$. An experienced examiner reviewed all results with the option to manually add or remove any incorrectly identified cells. The examiner then distinguished cones from the rods based on observation of cell brightness, size, and the presence (or absence) of an annulus surrounding the cones (the presence of which is a feature of cones in AO-SLO images). To investigate reliability of the experienced examiner's identification of cones and rods, a naive examiner (unfamiliar with cone and rod packing distributions) was asked to identify all cells (indiscriminant of type) in images from one subject.

\section{Distribution analysis}

Cone and rod density were calculated as the number of identified cells $/ \mathrm{mm}^{2}$. Images were scaled and pixels converted to $\mu \mathrm{m}$ based on axial length measurements using the method described by Bennett et $a l^{35}$ including their adjustment for different eccentricities. Cone-to-cone spacing was calculated as the mean distance from a given cone to its five nearest cone neighbors, averaged over all cones in the ROI (excluding those near the borders). Although hexagonal cone packing is expected to be observed at most locations (especially near the fovea), five nearest neighbors was chosen for this analysis so that regions of less dense packing would not skew the results. Rod-to-rod spacing was instead taken to be the mean distance to two nearest rod neighbors, because at more central eccentricities, where only a single ring of rods forms around each cone, rods may have only two adjacent rod neighbors. Voronoi analysis ${ }^{36}$ was performed using the locations of identified cones and rods to determine packing geometry in terms of number of nearest neighbors for each cell. Cone-to-all cell nearestneighbor results were based on Voronoi analysis using the positions of all identified cells, while cone-to-cone nearest-neighbor results used only the cone positions. Voronoi analysis of rod packing is complicated by the presence of gaps when cones are excluded. Hence, the number of rod-to-rod nearest neighbors was calculated as the number of rods within a cut-off radius of a given rod, averaged over all rods. This cut-off radius was taken to be $1.5 \times$ the rod spacing previously determined for each subject and retinal location.

\section{Results}

Figure 1 shows averaged, registered AO-SLO images spanning the range of retinal eccentricity from $30^{\circ} \mathrm{NR}$ to $30^{\circ} \mathrm{TR}$ for subject N1. Images are displayed with logarithmic intensity scaling to enhance visualization of the rod structure. In the foveal image, all cells are cones with a center-to-center spacing of $2.6 \pm 0.2 \mu \mathrm{m}$. At $3^{\circ} \mathrm{NR}$ and $3^{\circ} \mathrm{TR}$, the cones were noticeably larger compared with the fovea, and rods were observed between most adjacent cones. At larger eccentricities, both temporally and nasally, the cone density dropped markedly, while the rod density increased. In all images (aside from the fovea), the reflected signal from cones tended to be brighter and broader than that of rods, and they were typically surrounded by a distinct dark annulus. The images are similar in appearance to those seen in prior AO-SLO studies. ${ }^{19,20}$

Cones and rods were resolved in images from all subjects at all retinal locations, with the exception of $20^{\circ}$ NR for subjects N2 and N5 and $25^{\circ} \mathrm{NR}$ for subject N2. These data points were excluded from the subsequent analyses. The difficulty in imaging at $20-25^{\circ} \mathrm{NR}$ is attributed to the relatively thick nerve fiber layer at this area. ${ }^{37}$ Cone and rod densities as a function of retinal eccentricity for all five subjects are shown in Figure $2 \mathrm{a}$ and $b$, respectively. The symbols denote individual subject data with the solid black line indicating the mean. For comparison, the dashed black lines shows corresponding histological results. ${ }^{4}$ Mean cone density at the fovea was $164000 \pm 24000$ cones $/ \mathrm{mm}^{2}$ and decreased to values of $6700 \pm 1500$ and $5400 \pm 700$ cones $/ \mathrm{mm}^{2}$ at $30^{\circ}$ NR and $30^{\circ} \mathrm{TR}$, respectively. At high eccentricities (20$30^{\circ}$ ), cone densities in the NR averaged 39\% higher than the corresponding TR values. This was not the case at the central retina: from 3 to $10^{\circ} \mathrm{NR}$ and TR, cone density values varied by $<12 \%$. Rod density peaked at $25^{\circ} \mathrm{NR}$ $\left(124000 \pm 20000 \mathrm{rods} / \mathrm{mm}^{2}\right)$ and $20^{\circ} \mathrm{TR}(120000 \pm 12000$ rods $/ \mathrm{mm}^{2}$ ). Good agreement was seen between our AOSLO results and histology for both cone and rod densities, though our mean rod density values trended slightly lower $(\sim 12 \%)$ at retinal eccentricities beyond $5^{\circ}$. For the images assessed by the naive examiner (five locations from subject N1), cell density averaged $16 \%$ higher compared with those from the experienced examiner over the same image regions. This was attributed, in large part, to the common phenomenon of cones exhibiting side 

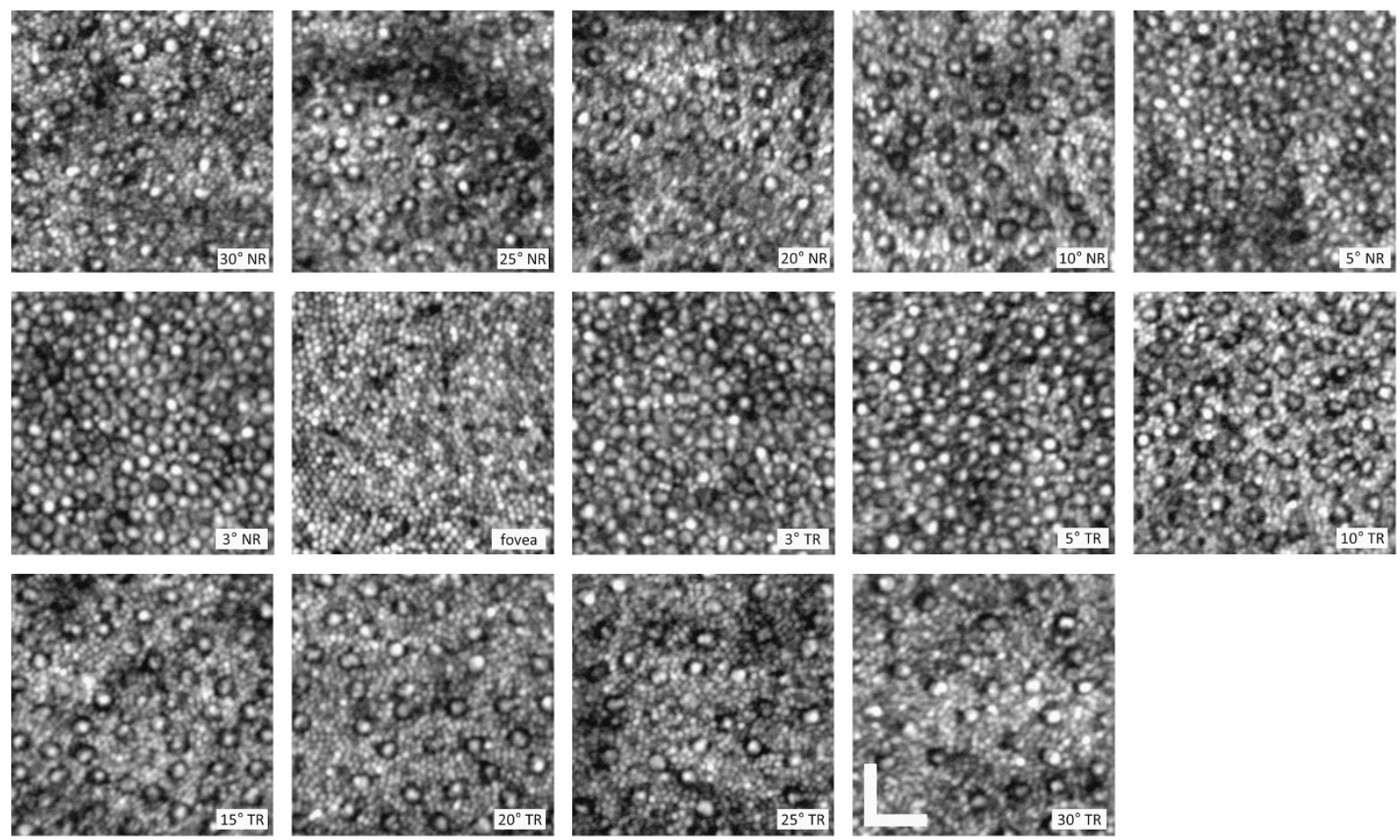

Figure 1 AO-SLO images of the cone and rod mosaic at locations spanning $30^{\circ}$ NR to $30^{\circ}$ TR for subject N1. Images are displayed with a logarithmic intensity scale to enhance the visualization of the rod photoreceptors. Each image is the registered average of $\sim 50$ frames. The scale bar is $25 \mu \mathrm{m}$.

lobes and the presence of faint intensity signals within the characteristic dark annulus, which the naive examiner often identified as separate cells.

Figure 2c and d shows results for cone and rod centerto-center spacing, respectively. Cone spacing increased from $2.7 \pm 0.2 \mu \mathrm{m}$ at the fovea to $14.6 \pm 1.4 \mu \mathrm{m}$ at $30^{\circ} \mathrm{NR}$ and $16.3 \pm 0.7 \mu \mathrm{m}$ at $30^{\circ} \mathrm{TR}$. In the NR, the rod spacing was lowest at $25^{\circ} \mathrm{NR}(2.1 \pm 0.1 \mu \mathrm{m})$. Temporally, the lowest rod spacing was at $20^{\circ} \mathrm{TR}(2.2 \pm 0.1 \mu \mathrm{m})$, beyond which it showed a statistically significant increase to $2.3 \pm 0.1 \mu \mathrm{m}$ at $30^{\circ} \mathrm{TR}(P<0.05)$. Figure 3 shows the ratio of rods to cones. The ratio was zero at the fovea since no rods are present. At both $3^{\circ} \mathrm{TR}$ and NR, rods

outnumbered cones roughly by $2: 1$. In the TR, the ratio increased sharply out to $25^{\circ}$, where rods outnumbered cones by $23: 1$, after which the ratio decreased. In the NR, the ratio continued to increase all the way to $30^{\circ}$ with 19 rods for every cone.

Figure 4 shows retinal images and corresponding Voronoi plots at three nasal retinal eccentricities from subject N5. The color coding of each cell domain corresponds to the number of nearest neighbors of either cell type. In the $10^{\circ} \mathrm{NR}$ and $30^{\circ} \mathrm{NR}$ plots, black dots denote the cells identified as cones. All cells in the foveal image are cones, and therefore this demarcation was not used. At the fovea, hexagonal cone packing was predominant with $55 \%$ of cones having six-sided domains, followed by $23 \%$ with five-sided, $19 \%$ with seven-sided, $2 \%$ with four-sided, and the remaining $1 \%$ had greater than seven-sided domains. At higher eccentricities, where both rods and cones are present, a more varied packing arrangement was observed. Cones exhibited greater spacing at $30^{\circ} \mathrm{NR}$ compared with $10^{\circ}$ NR, although they had slightly fewer nearest neighbors on average. At $10^{\circ} \mathrm{NR}, 31 \%$ of cones had 8 -sided domains, followed by $30 \%$ with 9 -sided, 30\% with $10-$ sided or more, and the remaining $9 \%$ having 7 or fewer sides. At $30^{\circ} \mathrm{NR}$, the corresponding values were 31, 33, 19 , and $17 \%$, respectively.

Figure 5 shows quantitative packing results for all subjects. Figure 5a shows the number of cone-to-all cell nearest neighbors as a function of retinal eccentricity obtained from the Voronoi analysis. At locations $5^{\circ}$ and beyond, where most cones are surrounded entirely by rods, this plot represents the average number of rods neighboring (forming a ring around) each cone. The fact that the number of neighbors decreased slightly beyond $20^{\circ}$ for both NR and TR appears to be a result of the increase in rod spacing (and presumably size) over these eccentricities. Figure $5 \mathrm{~b}$ shows the number of cone-tocone nearest neighbors obtained from Voronoi analysis that used only the cone positions (excluding rods). From 
a

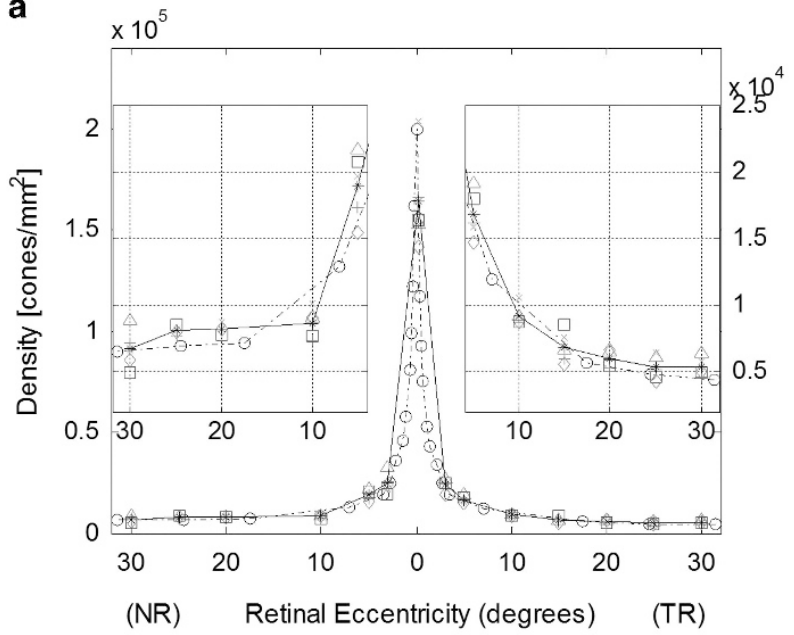

c

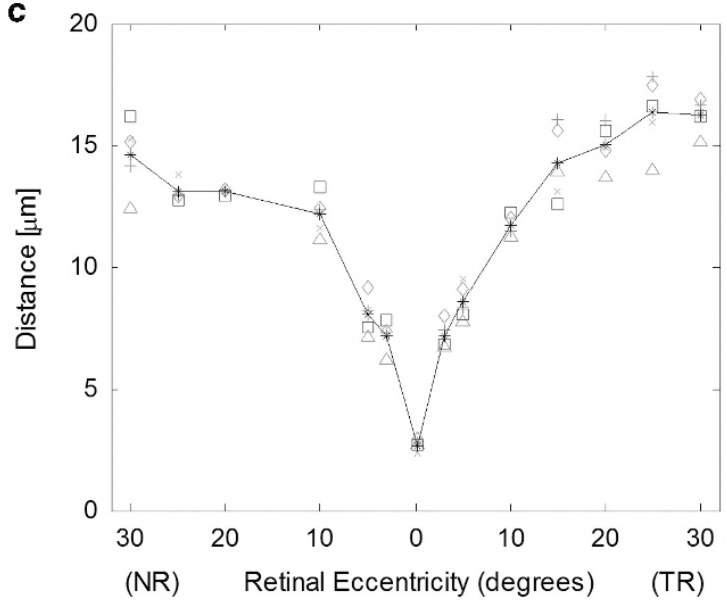

b

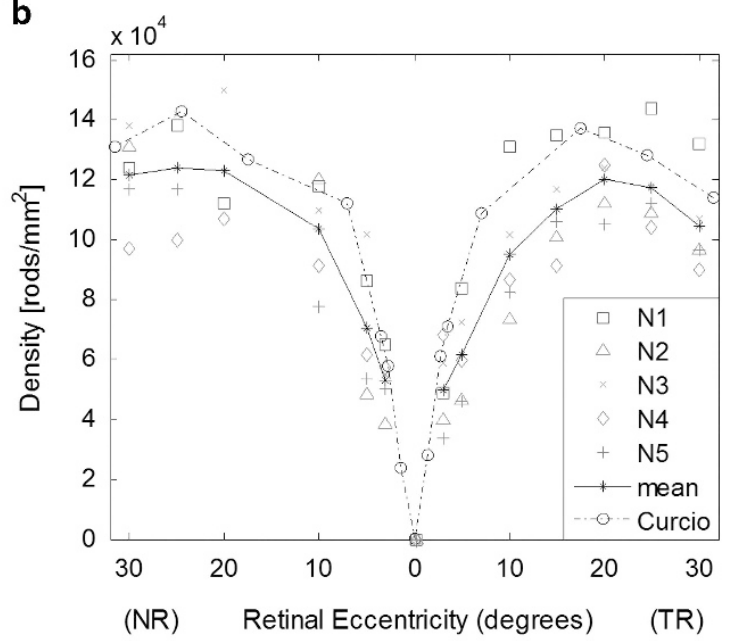

d

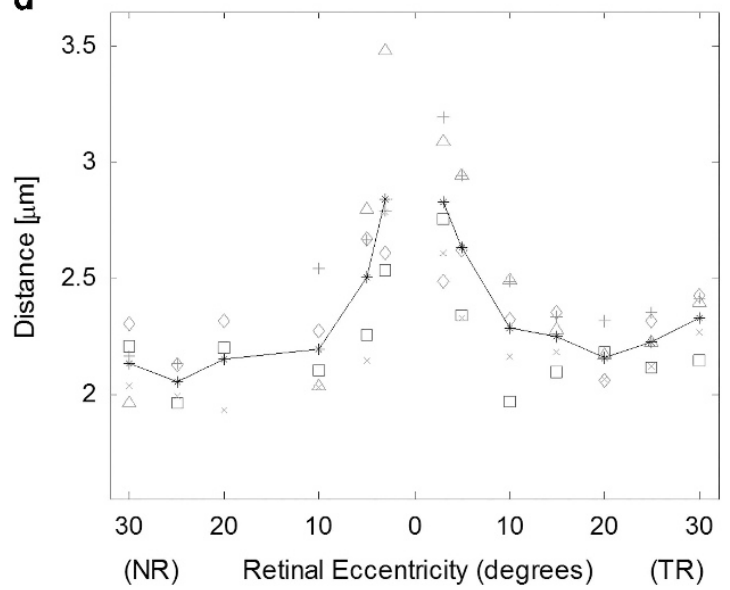

Figure 2 Photoreceptor density and spacing measurements for all five subjects as a function of retinal eccentricity. Results from individual subjects are denoted by the symbols, and the solid black line shows the mean. (a) Cone densities from the fovea to $30^{\circ} \mathrm{NR}$ and TR. The inset graphs show the $5-30^{\circ} \mathrm{NR}$ and TR data on expanded ordinate axes with scaling shown to the right; (b) rod density; (c) cone center-to-center spacing; and (d) rod center-to-center spacing. The black dashed lines in a and b show corresponding mean density results from Curcio et al ${ }^{4}$ assuming $1^{\circ}=290 \mu \mathrm{m}$.

this plot it is clear that, on average, cones are predominantly packed hexagonally at all retinal locations examined with slightly larger variation at larger eccentricities. Figure $5 \mathrm{c}$ shows the number of rod-to-rod nearest neighbors. The number of rod nearest neighbors was slightly higher in the TR than in the NR, plateauing at $3.9 \pm 0.1$ and $3.7 \pm 0.2$ neighbors, respectively. While many rods exhibited hexagonal packing (six neighbors) beyond $5^{\circ}$ from the fovea, a large number were adjacent to cones, which brought down the average number of nearest rod neighbors.

\section{Discussion}

The photoreceptor mosaics of five healthy subjects were imaged in vivo using an AO-SLO system, resolving both cones and rods over a $60^{\circ}$ horizontal span of retina ranging from $30^{\circ} \mathrm{NR}$ to $30^{\circ} \mathrm{TR}$. Although this is not the first study using an AO-SLO to characterize

photoreceptor packing, the range of eccentricities imaged has been doubled compared with prior work for both cones and rods. Furthermore, the results from the temporal retina show a decrease in rod density and an increase in rod spacing, implying an increase in rod size at eccentricities beyond $15^{\circ}$.

Overall, our results agreed well with the histology study by Curcio et al. ${ }^{4}$ Mean cone density (Figure 2a) followed the same trend across the retina, with results averaging $~ 3 \%$ higher than histology at locations excluding the fovea. The average peak cone density near the fovea of $164000 \pm 24000$ cones $/ \mathrm{mm}^{2}$ was lower than the histology finding of 199000 cones $/ \mathrm{mm}^{2}$, but this may be attributed to not precisely identifying the foveal pit in our image analyses. Our rod density measurements 
(Figure $2 \mathrm{~b})$ trended slightly lower $(\sim 12 \%)$ than Curcio's data but demonstrated similar behavior as a function of eccentricity. The mean rod spacing measured 2.0-2.5 $\mu \mathrm{m}$ at retinal locations $10^{\circ}$ and beyond (Figure $2 \mathrm{~d}$ ).

The Curcio study did not present quantitative cone and

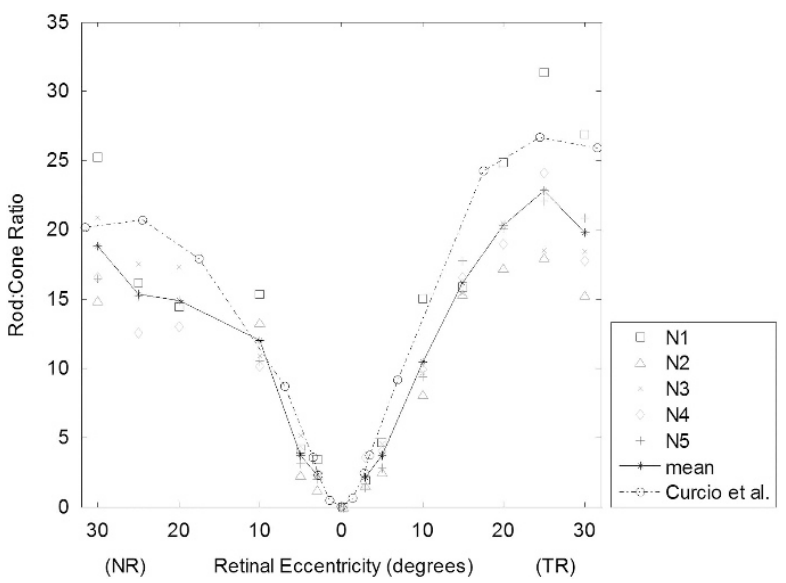

Figure 3 Rod-to-cone ratio as a function of retinal eccentricity for all subjects. Results from individual subjects are denoted by the symbols, and the solid black line shows the mean. The black dashed line shows the results from Curcio et al ${ }^{4}$ assuming $1^{\circ}=290 \mu \mathrm{m}$. rod spacing data; therefore, no histology comparison plots are given in Figure $2 \mathrm{c}$ and $\mathrm{d}$, but measurements on their single subject images suggest that the results presented here are in good agreement.

In a prior AO-SLO study, Dubra et al ${ }^{19}$ presented cone and rod density measurements at three temporal retinal eccentricities. Their rod density results also averaged slightly lower than Curcio's histology data at $10^{\circ}$ and $15^{\circ}$ TR but were in closer agreement at the $5^{\circ}$ TR location, and a similar trend was observed in this study. Another AO-SLO study by Merino et al ${ }^{20}$ examined cone and rod spacing nasally and inferiorly out to $\sim 12^{\circ}$. Compared with their results, our cone spacing values agreed well $(\sim 6 \%$ lower on average at $\left.3^{\circ}-10^{\circ} \mathrm{NR}\right)$, while our rod spacing results trended lower $\left(\sim 26 \%\right.$ lower at $5^{\circ}$ and $\left.10^{\circ} \mathrm{NR}\right)$.

The reason for lower rod density in this study compared with Curcio et $a l^{4}$ remains to be determined, though several issues may have a role. Specimen shrinkage in histological samples may be a potential explanation; however, a similar trend in our cone density results was not observed. Another possibility may be due to normal subject variability. This view is supported by noting that Curcio's mean values are within the range of our data at nearly all retinal locations. Differences in
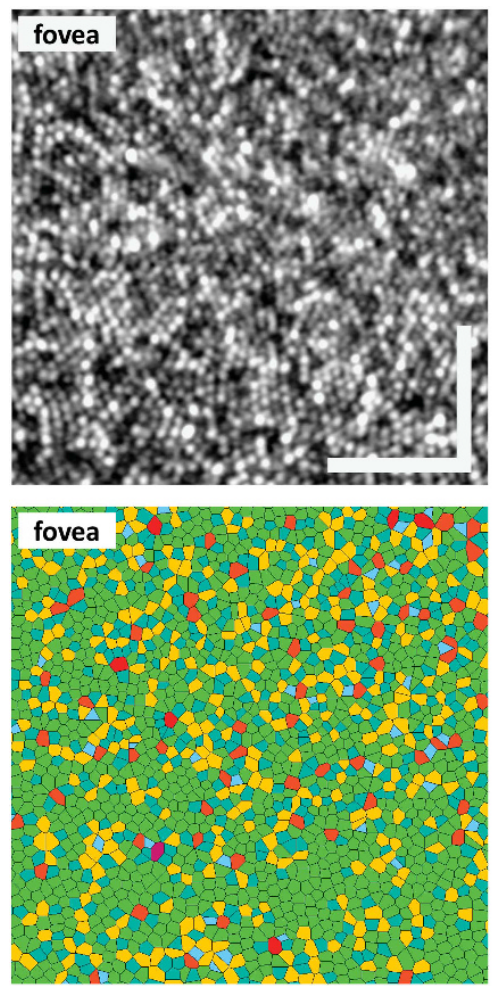
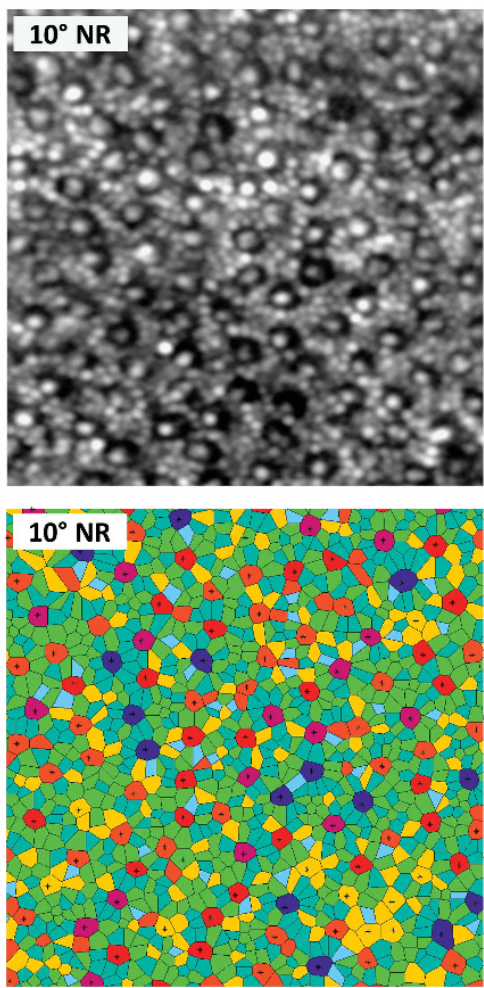
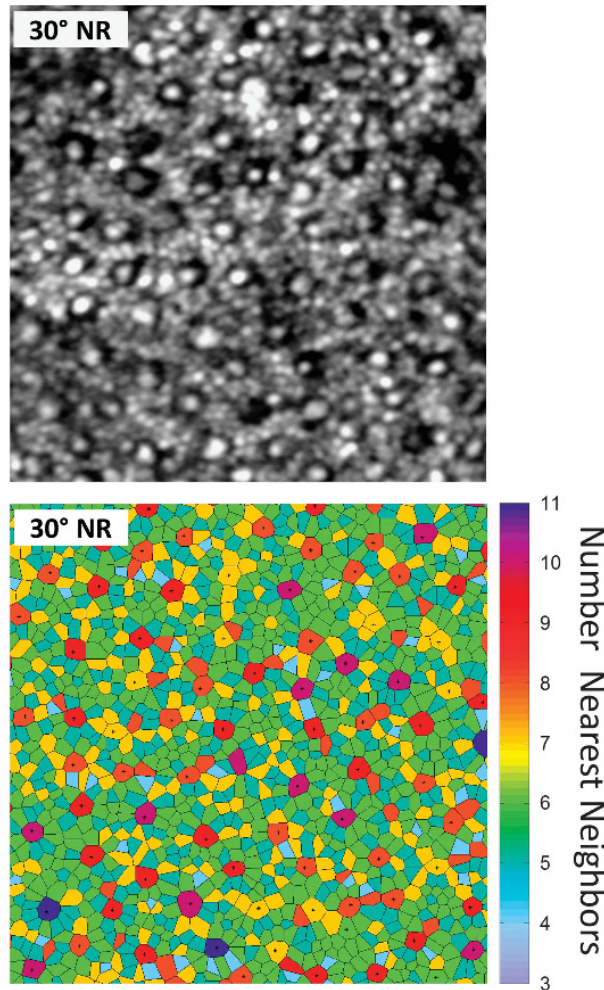

Figure 4 Photoreceptor mosaic images and corresponding Voronoi plots for the fovea, $10^{\circ} \mathrm{NR}$, and $30^{\circ}$ NR for subject N5. Each image is the registered average of $\sim 50$ frames, displayed with a logarithmic intensity scaling. For the Voronoi plots, the color coding of the cell domains indicate the number of neighboring cells (either cone or rod). Hexagonal packing (six nearest neighbors) is shown in green. In the $10^{\circ}$ and $30^{\circ} \mathrm{NR}$ Voronoi plots, cells identified as cones are marked with black dots. The scale bar is $50 \mu \mathrm{m}$. 

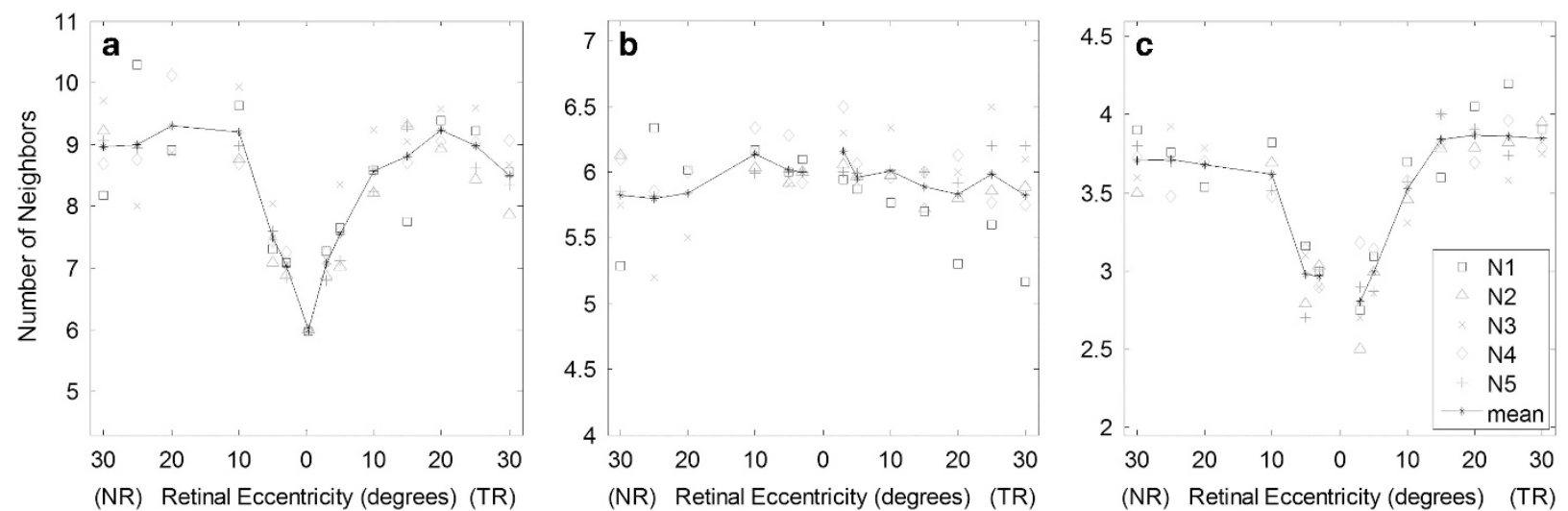

Figure 5 Quantitative cone and rod nearest neighbor results. (a) Number of nearest neighbors, cone-to-all cells, calculated from Voronoi analysis using all cell positions; (b) Number of nearest cone-to-cone neighbors (rods excluded); (c) Number of nearest neighbors, rod-to-rod, calculated as the number of rods within a radius defined by $1.5 \times$ rod spacing (as shown in Figure $2 \mathrm{~d}$ ). The solid black line shows the mean.

subjects' axial length, refractive error, and age between their population and ours may also be a factor. Finally, some rods may be missed during image analysis. In many images, there were instances where single identified rods appeared significantly brighter and often larger than neighbors. In such cases, it is possible that the feature in question may represent two bright rods located side-byside or a single bright rod obscuring neighboring cells. In addition, gaps in the rod mosaic were occasionally observed where the presence of a rod was expected (based on packing geometry), but no signal was observed. In this situation, it may be the case that a weakly reflecting rod was indeed present. Although such rods would not be counted in our AO-SLO images, they may be visible in histological images.

Prior AO studies have shown a decrease in cone density in myopic subjects, ${ }^{22,38}$ and a similar trend was observed here. The three emmetropic subjects had a mean SE of $-0.25 \mathrm{D}$, while for the myopes the mean SE was -3.1 D. Averaging the data from these two groups separately, lower cone and rod densities were found at nearly all retinal locations in the myopic group: $13 \%$ lower on average for cones and 19\% lower on average for the rods compared to the emmetropic group. Unlike in the earlier studies, which did not look beyond $12^{\circ}$, this trend extended into the mid-periphery for both cone and rod densities. Due to small sample sizes of the two refractive error groups statistical significance could not be established. However, these findings lend support to refractive error being a possible contributing factor to the differences observed between our rod density results and histology. ${ }^{4}$ Taking the three emmetropic subjects only, our results for rod density are in better agreement with histology ${ }^{4}$ being only $9 \%$ lower averaged over all retinal locations.
This study examined young subjects with large $(\sim 8 \mathrm{~mm})$ dilated pupils, good fixation, and clear media. Challenges will arise imaging older subjects, particularly when their pupils do not dilate fully. Differentiating between photoreceptor types in diseased eyes may also be complicated by the retinal condition.

In conclusion, we have successfully imaged cones and rods over a range of $60^{\circ}$ using AO-SLO, more than doubling the range of previous studies. We have demonstrated for the first time in vivo a decrease in rod density and an increase in rod spacing beyond $15^{\circ}$, implying that the rod size is increasing in the midperiphery. AO-SLO imaging has potential clinical significance, and the results here may serve as a benchmark for the detection and monitoring of retinal diseases where initial damage occurs in the periphery such as cone-rod dystrophy and RP.

\section{Summary}

What was known before

- Human cone and rod photoreceptor packing distributions have been determined via histology with a wide intersubjective variation.

- Adaptive optics $(\mathrm{AO})$ is a relatively recent advancement in imaging, which has allowed in vivo determination of rod and cone densities out to $15^{\circ}$ from the fovea.

\section{What this study adds}

- Demonstrates the ability to both image and measure cone and rod photoreceptors properties out to $30^{\circ}$ in both the nasal and temporal retina in vivo using $\mathrm{AO}$ scanning laser ophthalmoscopy.

- The ability to image in the mid-periphery will be significant for the study of retinal diseases such as retinitis pigmentosa.

- Show for the first time in vivo, an increase in the rod centerto-center spacing at the more peripheral retinal locations. 


\section{Conflict of interest}

The authors declare no conflict of interest.

\section{Acknowledgements}

This work was supported by National Institute of Health grant EY020901 and Department of Defense (DoD) Telemedicine and Advanced Technology Research Center (TATRC) grant W81XWH-10-1-0738.

\section{References}

1 Østerberg GA. Topography of the layer of rods and cones in the human retina. Acta Ophthalmol (Copenh) 1935; 13: $1-103$.

2 Polyak S. The Retina. University of Chicago Press: Chicago, 1941.

3 Farber DB, Flannery JG, Lolley RN, Bok D. Distribution patterns of photoreceptors, protein, and cyclic nucleotides in the human retina. Invest Ophthalmol Vis Sci 1985; 26 : 1558-1568.

4 Curcio CA, Sloan KR, Kalina RE, Hendrickson AE. Human photoreceptor topography. J Comp Neurol 1990; 292: 497-523.

5 Jonas JB, Schneider U, Naumann GO. Count and density of human retinal photoreceptors. Graefes Arch Clin Exp Ophthalmol 1992; 230: 505-510.

6 Hendrickson AE, Yuodelis C. The morphological development of the human fovea. Ophthalmology 1984; 91 603-612.

7 Dorey CK, Wu G, Ebenstein D, Garsd A, Weiter JJ. Cell loss in the aging retina. Relationship to lipofuscin accumulation and macular degeneration. Invest Ophthalmol Vis Sci 1989; 30: 1691-1699.

8 Curcio CA, Millican CL, Allen KA, Kalina RE. Aging of the human photoreceptor mosaic: evidence for selective vulnerability of rods in central retina. Invest Ophthalmol Vis Sci 1993; 34: 3278-3296.

9 Panda-Jonas S, Jonas JB, Jakobczyk-Zmija M. Retinal photoreceptor density decreases with age. Ophthalmology 1995; 102: 1853-1859.

10 Kimble TD, Williams RW. Structure of the cone photoreceptor mosaic in the retinal periphery of adult humans: analysis as a function of age, sex, and hemifield. Anat Embryol (Berl) 2000; 201: 305-316.

11 Liang J, Williams DR, Miller DT. Supernormal vision and high-resolution retinal imaging through adaptive optics. J Opt Soc Am A 1997; 14: 2884-2892.

12 Roorda A, Romero-Borja F, Donnelly W III, Queener H, Hebert T, Campbell M. Adaptive optics scanning laser ophthalmoscopy. Opt Express 2002; 10: 405-412.

13 Hermann B, Fernandez EJ, Unterhuber A, Sattmann H, Fercher AF, Drexler W et al. Adaptive-optics ultrahighresolution optical coherence tomography. Opt Lett 2004; 29: 2142-2144.

14 Miller DT, Qu J, Jonnal RS, Thorn KE. Coherence gating and adaptive optics in the eye. Proc SPIE 2003; 4956: 65-72.

15 Hartong DT, Berson EL, Dryja TP. Retinitis pigmentosa. Lancet 2006; 368: 1795-1809.
16 Birch DG, Anderson JL, Fish GE. Yearly rates of rod and cone functional loss in retinitis pigmentosa and cone-rod dystrophy. Ophthalmology 1999; 106: 258-268.

17 Szlyk JP, Fishman GA, Alexander KR, Peachey NS, Derlacki DJ. Clinical subtypes of cone-rod dystrophy. Arch Ophthalmol 1993; 111: 781-788.

18 Chui TY, Song H, Burns SA. Adaptive-optics imaging of human cone photoreceptor distribution. J Opt Soc Am A Opt Image Sci Vis 2008; 25: 3021-3029.

19 Dubra A, Sulai Y, Norris JL, Cooper RF, Dubis AM, Williams DR et al. Noninvasive imaging of the human rod photoreceptor mosaic using a confocal adaptive optics scanning ophthalmoscope. Biomed Opt Express 2011; 2: 1864-1876.

20 Merino D, Duncan JL, Tiruveedhula P, Roorda A. Observation of cone and rod photoreceptors in normal subjects and patients using a new generation adaptive optics scanning laser ophthalmoscope. Biomed Opt Express 2011; 2: 2189-2201.

21 Zhang T, Godara P, Blanco ER, Griffin RL, Wang X, Curcio CA et al. Variability in human cone topography assessed by adaptive optics scanning laser ophthalmoscopy. Am J Ophthalmol 2015; 160: 290-300.

22 Chui TY, Song H, Burns SA. Individual variations in human cone photoreceptor packing density: variations with refractive error. Invest Ophthalmol Vis Sci 2008; 49: 4679-4687.

23 Song H, Chui TY, Zhong Z, Elsner AE, Burns SA. Variation of cone photoreceptor packing density with retinal eccentricity and age. Invest Ophthalmol Vis Sci 2011; 52: 7376-7384

24 Rativa D, Vohnsen B. Analysis of individual conephotoreceptor directionality using scanning laser ophthalmoscopy. Biomed Opt Express 2011; 2: 1423-1431.

25 Miloudi C, Rossant F, Bloch I, Chaumette C, Leseigneur A, Sahel JA et al. The negative cone mosaic: a new manifestation of the optical stiles-crawford effect in normal eyes. Invest Ophthalmol Vis Sci 2015; 56: 7043-7050.

26 Doble N, Choi SS, Codona JL, Christou J, Enoch JM, Williams DR. In vivo imaging of the human rod photoreceptor mosaic. Opt Lett 2011; 36: 31-33.

27 Scoles D, Sulai YN, Langlo CS, Fishman GA, Curcio CA, Carroll J et al. In vivo imaging of human cone photoreceptor inner segments. Invest Ophthalmol Vis Sci 2014; 55: 4244-4251.

28 Song H, Rossi EA, Latchney L, Bessette A, Stone E, Hunter JJ et al. Cone and rod loss in stargardt disease revealed by adaptive optics scanning light ophthalmoscopy. JAMA Ophthalmol 2015; 133: 1198-1203.

29 Hansen SO, Cooper RF, Dubra A, Carroll J, Weinberg DV. Selective cone photoreceptor injury in acute macular neuroretinopathy. Retina 2013; 33: 1650-1658.

30 Godara P, Cooper RF, Sergouniotis PI, Diederichs MA, Streb MR, Genead MA et al. Assessing retinal structure in complete congenital stationary night blindness and Oguchi disease. Am J Ophthalmol 2012; 154: 987-1001.

31 Wells-Gray EM, Zawadzki RJ, Finn SC, Greiner C, Werner JS, Choi SS et al. Performance of a combined optical coherence tomography and scanning laser ophthalmoscope with adaptive optics for human retinal imaging applications. Proc SPIE 2015; 9335: 93350N1-93350N10.

32 Delori FC, Webb RH, Sliney DHAmerican National Standards I. Maximum permissible exposures for ocular safety (ANSI 2000), with emphasis on ophthalmic devices. J Opt Soc Am A Opt Image Sci Vis 2007; 24: 1250-1265. 
33 Stevenson SB, Roorda A. Correcting for miniature eye movements in high resolution scanning laser ophthalmoscopy. Proc SPIE 2005; 5688: 145-151.

34 Guizar-Sicairos M, Thurman ST, Fienup JR. Efficient subpixel image registration algorithms. Opt Lett 2008; 33: 156-158.

35 Bennett AG, Rudnicka AR, Edgar DF. Improvements on Littmann's method of determining the size of retinal features by fundus photography. Graefes Arch Clin Exp Ophthalmol 1994; 232: 361-367.
36 Aurenhammer F. Voronoi diagrams - a survey of fundamental geometric data structure. ACM Comput Surv 1991; 23: 345-405.

37 Varma R, Skaf M, Barron E. Retinal nerve fiber layer thickness in normal human eyes. Ophthalmology 1996; 103: 2114-2119.

38 Kitaguchi Y, Bessho K, Yamaguchi T, Nakazawa N, Mihashi T, Fujikado T. In vivo measurements of cone photoreceptor spacing in myopic eyes from images obtained by an adaptive optics fundus camera. Jpn J Ophthalmol 2007; 51: 456-461. 